\title{
GENERATION OF OPTICAL HARMONICS*
}

P. A. Franken, A. E. Hill, C. W. Peters, and G. Weinreich

The Harrison M. Randall Laboratory of Physics, The University of Michigan, Ann Arbor, Michigan

(Received July 21, 1961)

The development of pulsed ruby optical masers $\mathrm{s}^{1,2}$ has made possible the production of monochromatic (6943 A) light beams which, when focussed, exhibit electric fields of the order of $10^{5}$ volts $/ \mathrm{cm}$. The possibility of exploiting this extraordinary intensity for the production of optical harmonics from suitable nonlinear materials is most appealing. In this Letter we present a brief discussion of the requisite analysis and a description of experiments in which we have observed the second harmonic (at $\sim 3472 \mathrm{~A}$ ) produced upon projection of an intense beam of $6943 \mathrm{~A}$ light through crystalline quartz.

A suitable material for the production of optical harmonics must have a nonlinear dielectric coefficient and be transparent to both the fundamental optical frequency and the desired overtones. Since all dielectrics are nonlinear in high enough fields, this suggests the feasibility of utilizing materials such as quartz and glass. The dependence of polarization of a dielectric upon electric field $E$ may be expressed schematically by

$$
P=\chi E\left(1+\frac{E}{E_{1}}+\frac{E^{2}}{E_{2}{ }^{2}}+\cdots\right),
$$

where $E_{1}, E_{2} \ldots$ are of the order of magnitude of atomic electric fields ( $\left.\sim 10^{8} \mathrm{esu}\right)$. If $E$ is sinusoidal in time, the presence in Eq. (1) of terms of quadratic or higher degree will result in $P$ containing harmonics of the fundamental frequency. Direct-current polarizations should accompany the even harmonics.

Let $\vec{p}$ be that part of $\vec{P}$ which is quadratic in $\vec{E}$; that is, $\vec{p}$ is a linear function of the components of the symmetric tensor $\overrightarrow{\mathrm{E}} \overrightarrow{\mathrm{E}}$. The eighteen coefficients which occur in this function are subject to restrictions due to the point symmetry of the medium. These restrictions are, in fact, identical with those governing the piezoelectric coefficients. In particular, $\vec{p}$ necessarily vanishes in a material such as glass which is isotropic or contains a center of inversion. For crystalline quartz, however, there are two independent coefficients $\alpha$ and $\beta$ in terms of which

$$
\begin{aligned}
& p_{x}=\alpha\left(E_{x}{ }^{2}-E_{y}{ }^{2}\right)+\beta E_{y} E_{z}, \\
& p_{y}=-\beta E_{x} E_{z}-2 \alpha E_{x} E_{y}, \\
& p_{z}=0
\end{aligned}
$$

Table I. The square of the total $p$ perpendicular to the direction of propagation of light through crystalline quartz.

\begin{tabular}{cl}
\hline $\begin{array}{c}\text { Direction of incident } \\
\text { beam }\end{array}$ & $\begin{array}{l}\text { The square of the total } p \\
\text { perpendicular to direction } \\
\text { of propagation }\end{array}$ \\
\hline$x\left(E_{x}=0\right)$ & $p_{y}{ }^{2}+p_{z}{ }^{2}=0$ \\
$y\left(E_{y}=0\right)$ & $p_{z}{ }^{2}+p_{x}{ }^{2}=\alpha^{2} E_{x}{ }^{4}$ \\
$z\left(E_{z}=0\right)$ & $p_{x}{ }^{2}+p_{y}{ }^{2}=\alpha^{2}\left(E_{x}{ }^{2}+E_{y}{ }^{2}\right)^{2}$ \\
\hline
\end{tabular}

( $z$ is the threefold, or optic, axis; $x$ a twofold axis). If a light beam traverses quartz in one of the three principal directions, Eqs. (2) predict the results summarized in Table I. The secondharmonic light should be absent in the first case, dependent upon incident polarization in the second case, and independent of this polarization in the third.

If an intense beam of monochromatic light is focussed into a region of volume $V$, there should occur an intensity $I$ of second harmonic given (in Gaussian units) by

$$
I \sim\left(\omega^{4} / c^{3}\right)(p v)^{2}(V / v),
$$

where $\omega$ is the angular frequency of the second harmonic, $c$ the velocity of light, and $v$ an effective "volume of coherence"; that is, the size of a region within the sample in which there is phase coherence of the $p$ excitation. (This volume may in practice be much smaller than $V$.) An estimate of $v$ is governed by several considerations. For example, it is probably of no greater extent in the propagation direction than $\sim\left[n_{2}\right.$ $\left.\times\left(n_{2}-n_{1}\right)^{-1}\right] \lambda_{2}$, where $n_{1}$ and $n_{2}$ are the indices of refraction for the fundamental and second harmonic frequencies, respectively, and $\lambda_{2}$ is the wavelength of the second harmonic. The lateral extent of this volume is determined in large part by the coherence characteristics of the optical maser. The situation for a maser of the gas discharge ${ }^{3}$ type is clearly more favorable in this respect than that for the ruby device. ${ }^{1,2}$ For a coherence volume of $10^{-11} \mathrm{~cm}^{3}$, which we think may be realistic in our case, Eq. (1) indicates 


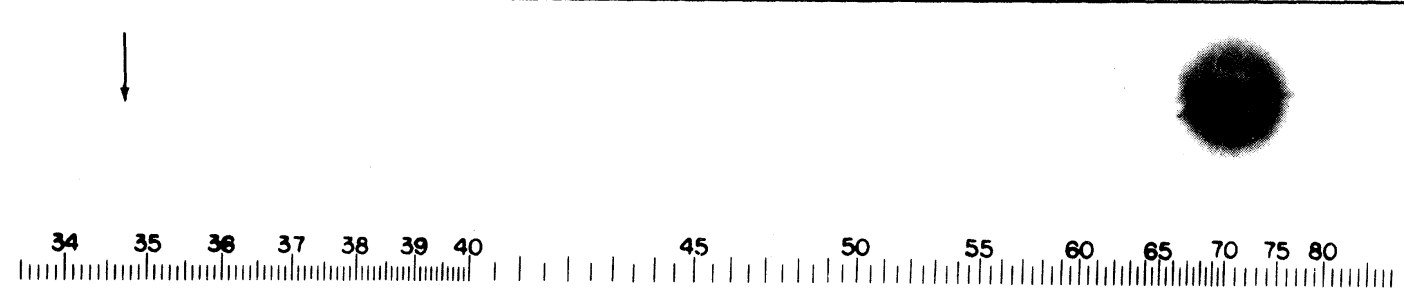

FIG. 1. A direct reproduction of the first plate in which there was an indication of second harmonic. The wavelength scale is in units of $100 \mathrm{~A}$. The arrow at $3472 \mathrm{~A}$ indicates the small but dense image produced by the second harmonic. The image of the primary beam at $6943 \mathrm{~A}$ is very large due to halation.

that second harmonic intensities as high as a fraction of a percent of the fundamental could be achieved.

In the experiments we have used a commercially available ruby optical maser ${ }^{4}$ which produces approximately 3 joules of $6943 \mathrm{~A}$ light in a onemillisecond pulse. This light is passed through a red filter for the elimination of the xenon flash background and is then brought to a focus inside a crystalline quartz sample. The emergent beam is analyzed by a quartz prism spectrometer equipped with red insensitive Eastman Type 103 spectrographic plates. A reproduction of the first plate in which there was an unambiguous indication of second harmonic (3472 A) is shown in Fig. 1. This plate was exposed to only one "shot" from the optical maser. We believe the following two facts, among others, rule out the possibility of artifact:

(1) The light at $3472 \mathrm{~A}$ disappears when the quartz is removed or is replaced by glass.

(2) The light at 3472 A exhibits the expected dependence on polarization and orientation summarized in Table I.

Considerations of the photographic image density and the efficiency of the optical system lead us to believe that the order of $10^{11}$ second harmonic photons were generated within the quartz sample per pulse.

The production of a second harmonic should be observable in isotropic materials such as glass if a strong bias field were applied to the sample. This bias could be oscillatory, thus producing sidebands on the fundamental frequency and the harmonics.

We would like to thank the staff of Trion Instruments, Inc., for their valuable and sustained cooperation in this work.

\footnotetext{
${ }^{*}$ This work was supported in part by the U. S. Atomic Energy Commission.

${ }^{1}$ T. H. Maiman, Nature $\underline{187}, 493$ (1960).

${ }^{2}$ R. J. Collins et al., Phys. Rev. Letters $\underline{5}, 303$ (1960).

${ }^{3}$ A. Javan, W. R. Bennet, and D. R. Herriott, Phys. Rev. Letters 6 , 106 (1961). Even though the intensity of the gas device is very low compared with ruby masers, the gain in coherence volume and the potential improvement of focussing suggest that the gas maser may be comparable or even superior as a source for optical harmonics.

${ }^{4}$ Trion Instruments, Inc., Model No. TO-3000.
} 


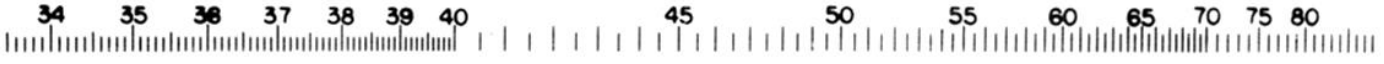

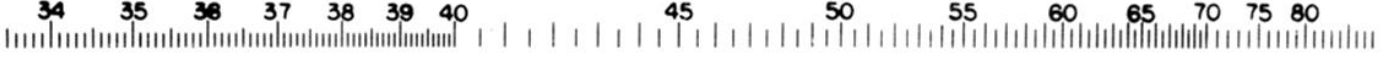

FIG. 1. A direct reproduction of the first plate in which there was an indication of second harmonic. The wavelength scale is in units of $100 \mathrm{~A}$. The arrow at $3472 \mathrm{~A}$ indicates the small but dense image produced by the second harmonic. The image of the primary beam at $6943 \mathrm{~A}$ is very large due to halation. 